\title{
Mécanismes de Bricard : Quelles applications dans un système déployable?
}

\author{
Chloé Lefeu ${ }^{1, *}$, Margaux Gillet ${ }^{1}$, et Marc Mimram ${ }^{1}$ \\ ${ }^{1}$ Ecole d'Architecture, de la Ville et des Territoires de Marne-la-Vallée, 77420 Champs-sur-Marne, \\ France
}

\begin{abstract}
Résumé. Les mécanismes de Bricard sont des mécanismes complexes et les recherches précédentes sur le sujet sont principalement basées sur leur compréhension mathématique et l'analyse de leur mouvement. La matérialisation physique du mécanisme de Bricard, ses applications et les connections possibles entre plusieurs mécanismes ne sont encore qu'à leurs débuts. Plusieurs utilisations de ces mécanismes sont étudiées dans le domaine des structures déployables à échelle humaine. Des choix de matériaux et de processus de fabrication sont discutés.
\end{abstract}

Mots-clés. Mécanisme de Bricard, structure déployable, mécanisme structurel, mécanisme reconfigurable, anneau de tétraèdres.

\begin{abstract}
Bricard linkages are complex mechanisms, and previous research on the subject was mainly based on the mathematical and kinetic analysis of its behavior. Thence, the physical materialization of Bricard linkages, its applications and the possible connections between several linkages are at an early stage. Several uses of such mechanisms are studied in the field of deployable structure at human scale. Choices of materials and fabrication process are discussed.
\end{abstract}

Keywords. Bricard linkages, deployable structure, structural mechanism, reconfigurable mechanism, ring of tetrahedra.

\section{Introduction}

En 1897, Raoul Bricard, ingénieur et mathématicien français, publie un article nommé " Mémoire sur la théorie de l'octaèdre articulé » dans le Journal de mathématiques pures et appliquées 5e série (Bricard, 1897). C'est dans cet essai que sont caractérisés, pour la première fois, les mécanismes qui prendront son nom par la suite. Les recherches qui y sont menées font suite à une problématique soulevée par M. C. Stephanos : "existe-t-il des polyèdres à faces invariables susceptibles d'une infinité de transformations avec altération seulement des angles solides et des dièdres? ». Raoul Bricard propose alors une solution générale à ce problème dans le cas d'octaèdres à faces triangulaires. Il caractérise ainsi trois cas d'octaèdres déformables, puis trois cas de mécanismes surcontraints à six liaisons

\footnotetext{
${ }^{*}$ Corresponding author: chloe.lefeu@gmail.com
} 
rotoïdes. De tels mécanismes sont définis par le critère de Kutzbach comme des structures rigides.

Cependant, les mécanismes de Bricard ont un degré de liberté. Cette mobilité leur donne le nom de mécanismes paradoxaux, ou surcontraints. Ce sont aussi les seuls mécanismes à six liaisons rotoïdes (6R) qui ne soient pas des modifications de 4R ou 5R. Ces propriétés les rendent intéressants à étudier pour les mathématiciens et ingénieurs. La surcontrainte des mécanismes de Bricard (6R) et de Bennet (4R) les rendent attrayants pour le développement de structures déployables. Une fois leur unique degré de liberté bloqué, ces mécanismes deviennent en effet des structures hyperstatiques. G.T. Bennett est le premier à étudier la géométrie des octaèdres déformables et leurs propriétés cinétiques (Bennett, 1903). En 1980, J.E. Baker donne les équations de fermeture des six mécanismes de Bricard (Baker, 1980).

Le travail présenté dans la section 4.2 a été réalisé en collaboration avec Valentin Batlle, Aurélien Beck, Julien Glath et Brieuc Graillot dans le cadre de notre thèse de Master à l'École d'Architecture de la Ville et des Territoires de Marne-la-Vallée.

\section{Threefold-symmetric Bricard linkages}

\subsection{Histoire et définition}

En 2003, W.W. Gan et S. Pellegrino (2003) ont présenté un nouveau type de structures déployables, qui forment une boucle fermée lorsqu'elles sont déployées et qui se regroupent en un paquet de barres lorsqu'elles sont repliées. Elles se composent de quatre ou six barres connectées les unes aux autres par des liaisons rotoïdes. De telles structures ont été précédemment présentées à la NASA par (Crawford et al., 1973). Le système est adapté et utilisé pour la poutre de rive d'une antenne dépliable.

A la suite de leur article, Chen et al. (2005) ont investigué les utilisations possibles de ces mécanismes pour les structures déployables. En 2005, ils définissent un nouveau type de liaison Bricard, nommé «threefold-symmetric Bricard linkages », lien Bricard à triple symétrie.

Ce mécanisme possède deux caractéristiques principales : il a toujours trois plans de symétrie et les deux arêtes d'un tétraèdre qui le relie à ses voisins sont perpendiculaires. Ces propriétés en font un mécanisme de Bricard particulier, puisqu'il répond simultanément aux critères de définition d'un mécanisme de Bricard à symétrie axiale et à ceux d'un mécanisme orthogonal de Bricard. Si les angles de torsion ne sont pas égaux à $\pi / 2$, le mécanisme est toujours mobile. Seule la condition de triple symétrie définit les threefoldsymmetric Bricard linkages; les angles perpendiculaires en font un cas particulier.

Il peut être défini par

$$
\begin{array}{rcc}
\mathrm{a}_{12}=\mathrm{a}_{23}=\mathrm{a}_{34}=\mathrm{a}_{45}=\mathrm{a}_{56}=\mathrm{a}_{61}=1 & \mathrm{R}_{\mathrm{i}}=0 \quad \text { pour tout } \mathrm{i} \\
\alpha_{12}=\alpha_{34}=\alpha_{56}=\alpha & \alpha_{23}=\alpha_{45}=\alpha_{61}=360^{\circ}-\alpha
\end{array}
$$

avec :

$\mathrm{a}=$ longueur des barres reliant les liaisons rotoïdes

$\mathrm{R}=$ vecteur directeur des barres

$\alpha=$ angle de torsion entre les axes de deux liaisons consécutives 


\subsection{Cinématique}

Parallèlement à la publication de Gan et Pellegrino (2003), Chen a publié sa thèse de doctorat (Chen, 2003 ; Chen et al., 2005), où elle étudie la conception de mécanismes structurels. La première partie est consacrée aux mécanismes de Bennett, la seconde aux mécanismes de Bricard, et plus particulièrement au threefold-symmetric Bricard linkage. Elle y étudie les variations de $\varphi$ et de $\theta$ en fonction d' $\alpha$, c'est-à-dire la fluidité de rotation du mécanisme. Le même comportement est observé pour les angles $\alpha$ et $\pi$ - $\alpha$.

Nous observons que toutes les lignes passent par les points $(0,2 \pi / 3),(0,-2 \pi / 3),(2 \pi / 3$, $0),(-2 \pi / 3,0)$. Cela veut dire que tous les threefold-symmetric Bricard linkages peuvent être aplatis en un triangle équilatéral planaire. Lorsque $0 \leq \alpha<\pi / 3$ ou $2 \pi / 3<\alpha \leq \pi$, le mouvement n'est pas continu; il est bloqué physiquement par l'intersection de trois liaisons. Le mécanisme forme une boucle fermée au mouvement fluide pour $\pi / 3<\alpha \leq 2 \pi / 3$. L'angle de torsion $\alpha$ doit donc être la conséquence d'un choix quant à l'usage du mécanisme : pour une structure déployable telle qu'une antenne spatiale, il est important de trouver une position finale bloquée au mouvement du mécanisme. Au contraire, pour certaines applications comme celles présentées ci-dessous, il est intéressant de garder cette continuité du mouvement.

Chen a pour sa part développé dans sa thèse le cas de $\alpha=\pi / 3$ ou $\alpha=2 \pi / 3$. Les angles $\varphi$ et $\theta$ atteignent $\pi$ ou $-\pi$ simultanément, ce qui correspond à la position la plus compacte. Ce sont les seuls angles qui proposent cette position compacte.

\section{Etat de l'art : une matérialisation à ses débuts}

\subsection{Construire des mécanismes de Bricard}

Afin de tester ses hypothèses, Chen a réalisé plusieurs prototypes dans des matériaux divers. Ses expérimentations mettent en avant un point crucial de la matérialisation des mécanismes de Bricard : la rigidité relative des liaisons rotoïdes et des faces des tétraèdres. Les rotules doivent pouvoir subir une légère déformation plastique pour effectuer un mouvement fluide. Si les faces sont moins rigides que les liaisons, ils vont reprendre les charges exercées sur le mécanisme.

Luo et al. (2007) ont étudié les applications des threefold symmetric Bricard linkages dans le domaine des structures rétractables, pour un toit par exemple. Au contraire de Yan Chen, qui a matérialisé les éléments entre les rotules par des tubes à section triangulaires, Luo et ses collèges les ont réalisés sous la forme de tétraèdres. Cette conception apporte une notion d'opacité et d'étanchéité qui n'est pas présente dans les précédentes études. La fabrication des éléments sous forme de volumes leur donne en outre plus d'inertie. Le prototype qu'ils ont réalisé est composé de faces en plastique et de charnières métalliques installées entre les faces internes des tétraèdres. Les liaisons ont donc ici plus de rigidité que les faces, et ne risquent pas de subir des déformations.

\subsection{Kinetogami}

Un anneau de tétraèdres réguliers peut être réalisé à partir d'une feuille de papier. Concilier un pliage 3D avec un mode de fabrication 2D pour créer des mécanismes ayant plusieurs degrés de liberté et la capacité de se déployer est aujourd'hui un champ de recherches spécifiques, nommé Kinetogami par ses inventeurs, Gao et al. (2013). Cette dénomination regroupe le mot grec «Kinetikos » et le mot japonais « kami », signifiant littéralement que des mécanismes polyédraux et des structures en mouvements sont créés à 
partir d'une simple feuille de papier. Les liaisons pliées relient des volumes creux et non des tiges pleines. Cette cavité intérieure au mécanisme peut accueillir différentes fonctions, comme nous le verrons plus tard. Les auteurs proposent plusieurs configurations à partir de plusieurs tétraèdres. L'une d'elles, la «Multi-Serial-Loops » se présente sous la forme d'un hexagone en position fermée et sous celle d'un hexagramme évidé en position ouverte.

Ces recherches ont permis la fabrication d'un robot «HexaMorph » (Gao et al., 2014) dont la structure est réalisée à partir d'une planche de carton, ce qui est à la fois léger et économique. Contrairement à d'autres systèmes robotiques modulaires, l'HexaMorph ne possède pas de plateforme centrale, et sa structure lui permet de bouger continuellement jusqu'à se retourner. Le robot peut être positionné selon différentes configurations sans avoir à le démonter. Il est aussi très facile à construire puisque l'assemblage et la fabrication sont réalisés en $2 \mathrm{D}$, le pliage et la reconfiguration en 3D. L'intérieur des tétraèdres est utilisé pour stocker les composants électroniques provoquant son mouvement. Ils sont contrôlés à distance par un ordinateur. D'autres matériaux peuvent être utilisés, s'ils répondent à certains critères : les faces doivent être rigides et les liaisons flexibles. Les impressions 3D permettent aujourd'hui de répondre à de telles demandes. Les faces du prototype sont réalisées à partir de polypropylène.

\section{Du modèle mathématique à sa réalisation physique}

\subsection{Définir les enjeux de la conception}

\subsubsection{Expérimentations manuelles}

C'est à travers le modèle de chaîne de tétraèdres réguliers que nous avons approché en premier lieu les mécanismes de Bricard. Nous avons commencé à étudier empiriquement des chaînes de six tétraèdres, autrement qualifiées de kaléidocycles. Nous les avons réalisées par pliage d'une seule feuille de papier ; ces objets laissent voir alternativement les quatre faces des tétraèdres réguliers d'un même point de vue. Ils n'offraient cependant aucune position notable dans le cycle de rotation; seules les couleurs des faces permettaient de les différencier.

Dans l'optique d'une utilisation en tant qu'élément architectural, le patron des tétraèdres a été modifié afin de présenter des variations dans l'ouverture du module. Les quatre faces en triangles isocèles sont remplacées par deux faces en triangles rectangles et deux faces en triangle isocèle (figure 1a). Cette modification apporte quatre phases notables lors de la rotation du mécanisme, représentées figure $1 \mathrm{~b}$ : une position fermée, une position intermédiaire, une position ouverte et une deuxième position intermédiaire qui permet de boucler jusqu'à la position fermée.
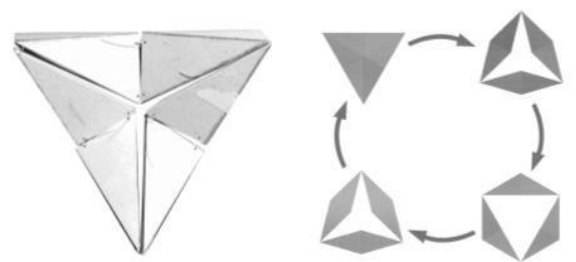

Figure 1. (a) Premier prototype de kaléidocycle, (b) Phases de la rotation.

Les utilisations possibles d'un tel dispositif sont variées. Il pourrait être utilisé comme toiture dont l'ouverture varie selon les conditions météorologiques (soleil, intempéries), un mur dans lequel peut s'ouvrir une entrée, ou à une plus petite échelle, du mobilier. Même si 
le mécanisme en lui-même peut effectuer sa rotation indéfiniment, cette mobilité peut être restreinte par le dispositif externe qui provoque la rotation par exemple.
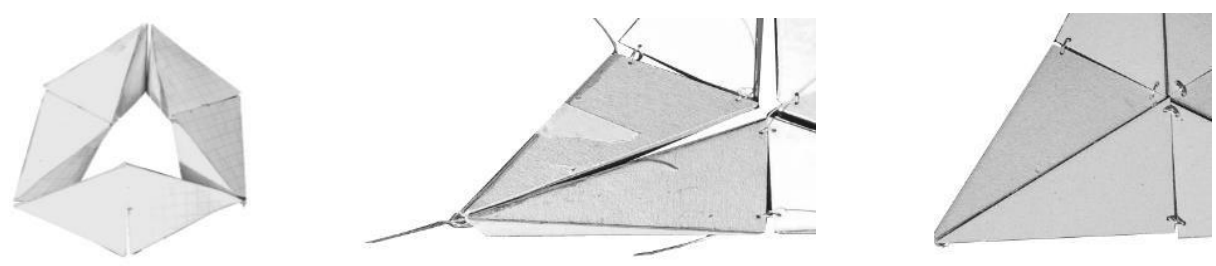

Figure 2. (a) Prototype en papier, (b) Liaisons en fil, (c) Liaisons métalliques.

La conception du modèle théorique présenté ci-dessus induit des liaisons rotoïdes parfaites et des faces de tétraèdres sans épaisseur. Un modèle en papier répond à ces prérequis, et est intéressant pour une étape de form finding / use finding, avec une hauteur des mécanismes de 5 centimètres (voir figure 2a). Ce modèle peut difficilement s'adapter à une plus grande échelle, puisque les faces et liaisons en papier perdent alors leur rigidité. Ce changement d'échelle est par ailleurs important pour interroger la fabrication et la matérialité des liaisons qui sont des éléments cruciaux du design des mécanismes. La deuxième session de prototypes fut réalisée avec du carton $3 \mathrm{~mm}$ et du fil de pêche pour les liaisons (voir figure $2 \mathrm{~b}$ ). Ces prototypes mesurent $20 \mathrm{~cm}$ de haut, ce qui permet de tester la nature des liaisons et d'étudier l'assemblage entre plusieurs mécanismes de Bricard. L'utilisation de fil de pêche n'était pas satisfaisante, ce matériau étant trop flexible pour ne permettre qu'une rotation pure autour de l'axe central de la liaison. Il fut remplacé par des anneaux métalliques qui améliorèrent le dispositif (voir figure 2c). Le matériau des tétraèdres -le carton- n'est pas approprié pour un usage dynamique du mécanisme, puisqu'il s'use très vite si le mécanisme est testé en rotation. De plus, la disposition des liaisons n'est pas optimale : leur épaisseur crée des conflits entre eux durant la rotation.

\subsubsection{Paver le plan}

De par la forme du mécanisme de Bricard définie ci-dessus, il est possible de paver le plan avec des modules en position ouverte et en position fermée. Le mouvement de chaque mécanisme dépendant de sa liberté de rotation autour de leur propre centre, la création d'un pavage où les mécanismes sont liés par leurs liaisons les rend interdépendants. Une seule immobilisation est alors suffisante pour bloquer le mouvement de tout le réseau. Il est aussi possible de contreventer le réseau de mécanisme en en installant un deuxième par-dessus en quinconce (figure 3a). Ce type de disposition peut être utilisé comme claire-voie (figure 3b), mais le mouvement du réseau est alors totalement bloqué.

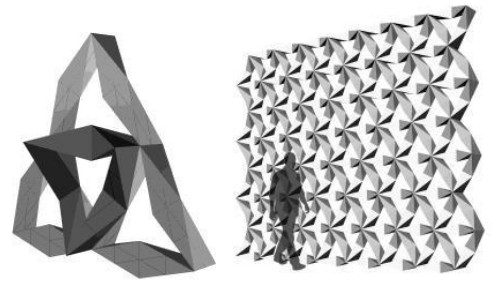

Figure 3. (a) Disposition en quinconce, (b) Exemple d'application. 


\subsubsection{Du plan à la voûte}

Un second prototype de pavage est assemblé avec des modules tous en position fermée. Comme dans la section précédente, les liaisons sont réalisées avec des anneaux métalliques. Chaque liaison est commune à trois modules, donc à six tétraèdres. À un mécanisme de Bricard central, trois lui sont attachés, un à chaque côté. Cet ensemble constitue un nouveau module, auquel sont connectés trois autres de ces modules de la même manière que pour leurs sous-modules (figure 4a). La figure créée est en forme de triangle. Lorsque des forces sont appliquées à ce nouveau mécanisme, il se courbe hiérarchiquement. Les plus grands modules s'éloignent les uns des autres ; ce mouvement global est transmis aux mécanismes de Bricard qui les composent et qui effectuent leur propre rotation. Cette hiérarchie dans le mouvement est une conséquence du processus de fabrication. Si toutes les liaisons des mécanismes étaient communes à au moins deux d'entre eux, aucun mouvement ne serait possible, les connections contreventant l'ensemble.

L'assemblage présenté ci-avant ne peut plus effectuer une rotation de $360^{\circ}$, il est restreint à $180^{\circ}$ maximum par le conflit physique de ses propres éléments (figure $4 \mathrm{~b}$ ). Il peut cependant être utilisé comme toiture ou ombrière. C'est l'éloignement ou le rapprochement des trois angles de l'assemblage qui permet de faire bouger les liaisons internes par leur unique degré de liberté. Si ce mouvement est bloqué, ce degré de liberté l'est aussi. Ainsi, fixer au sol ces angles peut empiriquement transformer le mécanisme global en structure.
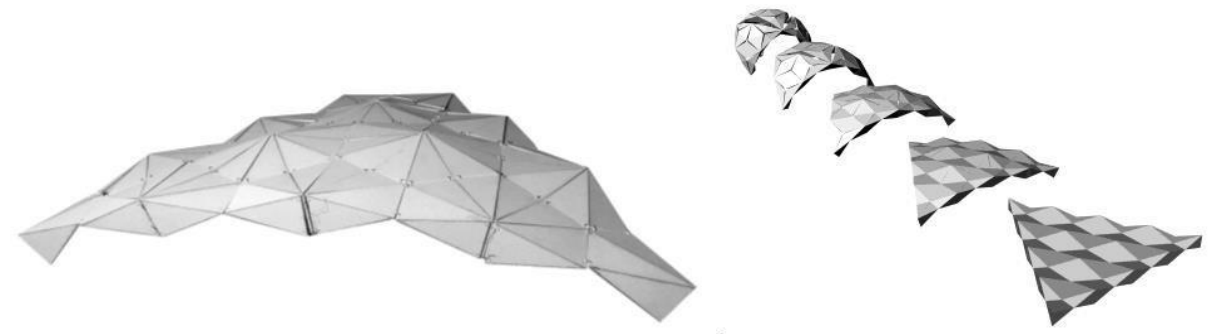

Figure 4. (a) Assemblage de 16 kaléidocycles, (b) Mouvement de l'assemblage.

\subsection{Kinésis, un prototype à l'échelle humaine}

\subsubsection{Augmenter l'échelle du modèle}

Basé sur les précédents travaux et recherches, nous avons conçu un nouveau prototype d'une échelle beaucoup plus importante, puisque le mécanisme de Bricard mesure 1 mètre de haut. Cela nous permet de travailler avec beaucoup plus de finesse sur les assemblages. Nous avons décidé à cette échelle de représenter les tétraèdres par leurs arêtes et non par leurs faces (figure 5a). En effet, la rigidité des faces nécessaire à cette échelle est trop contraignante : le mécanisme est alors trop lourd pour pouvoir être testé aisément. Nous avons, dans un premier temps, réalisé un prototype de tétraèdre avec des arêtes en bois, mais ce matériau n'est pas intéressant pour cet usage : il est trop fragile à cette échelle, et les assemblages entre plusieurs arêtes sont complexes. Nous avons alors testé un assemblage de tubes creux en aluminium. La modélisation sur Rhinocéros et Grasshopper d'un mécanisme de Bricard facilite la transition du modèle numérique au modèle physique : une étape intermédiaire est introduite, où les éléments du modèle physique sont modélisés avant d'être fabriqués. Ainsi, nous avons pu concevoir des nœuds imprimables en 3D répondant à nos besoins spécifiques : assembler les trois tubes en aluminium à chaque angle. 
Contrairement au travail personnel présenté ci-dessus et aux expérimentations de Yaozhi et ses collèges, nous avons ici décidé de placer les liaisons vers l'extérieur du système. Chaque rotule est maintenant créée par un tube en aluminium de chaque tétraèdre, auquel est collé un connecteur en plexiglas, qui tourne librement autour d'un troisième tube d'aluminium qui est l'axe de rotation de la liaison (figures $5 \mathrm{~b}$ et $5 \mathrm{c}$ ). Cette nouvelle disposition est intéressante puisque les tétraèdres peuvent être préfabriqués, et reliés ensemble plus tard. Ce système est aussi amovible, flexible et rend possible l'assemblage de plusieurs mécanismes de Bricard sur une même liaison.

La conception d'une maquette numérique a permis à cette étape d'utiliser toutes les potentialités des fabriques numériques : découpe laser pour les connecteurs des liaisons en plexiglas, et impression 3D pour les connecteurs liant les tubes en aluminium. Ces outils, qui ont respectivement une précision de 0,1 à $0,2 \mathrm{~mm}$ et de $0,025 \mathrm{~mm}$, permettent d'approcher au mieux le modèle théorique parfait. La précision de 0,6 $\mathrm{mm}$ sur l'épaisseur des tubes en aluminium semble alors grossière lorsqu'elle est comparée aux éléments précédemment cités.
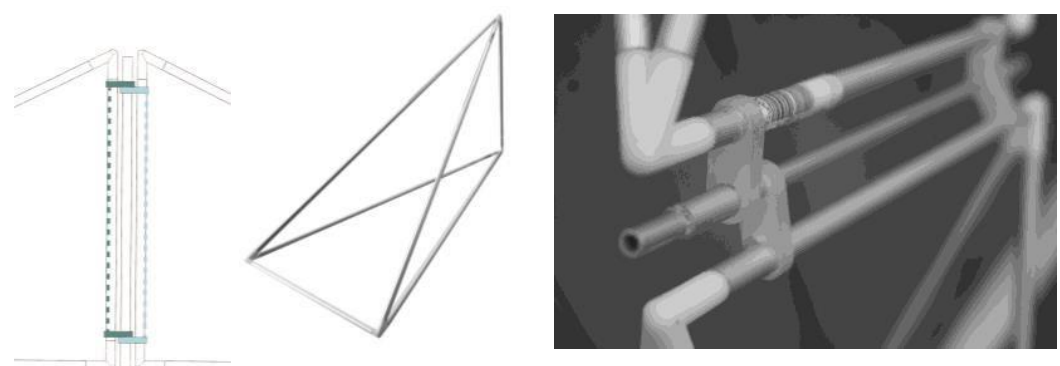

Figure 5. (a) Schéma des jonctions, (b) et (c) Photographies des tétraèdres.

\subsubsection{Kinésis, un pavillon expérimental}

L'organisation présentée dans la section 3.2 est intéressante puisqu'elle peut effectuer une rotation de $360^{\circ}$ et propose ainsi plusieurs dispositions singulières (figure 6). Nous avons donc décidé de concevoir plusieurs «objets » suivant cette disposition, avec les modules hauts d'un mètre présentés ci-dessus. Ces objets sont nommés «couronnes» d'après leur forme.
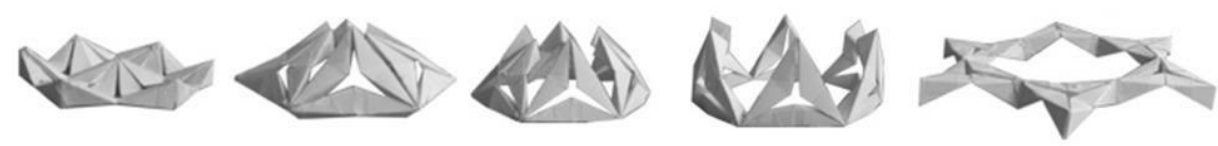

Figure 6. Phases de la rotation.

La modélisation sur Catia d'une couronne nous a permis de voir que la discrétisation de chaque mécanisme de Bricard en éléments finis a créé un conflit entre chaque module lors de la rotation au centre. En effet, les liaisons entre tétraèdres sont excentrées sur notre modèle ; les angles libres de chaque mécanisme de Bricard se bloquent physiquement lors de leur rencontre au centre. Pour y remédier, les liaisons communes à deux mécanismes de Bricard sont encore plus externalisées. Ainsi, le centre de chaque couronne n'est la position exacte d'aucune arête, elles sont toutes situées autour de ce centre lorsque la couronne est fermée. Cette modification crée des changements dans le modèle, puisque les longueurs entre deux liaisons successives ne sont alors plus égales. Cependant, cette imperfection n'est pas assez significative sur la totalité du modèle pour en empêcher le bon fonctionnement.

La suspension d'une couronne offre des possibilités spatiales intéressantes : la couronne est consécutivement une toiture pleine, un espace circulaire délimité par des mécanismes 
verticaux et une ombrière dont le centre est vide d'éléments. Cette disposition est par ailleurs intéressante puisqu'elle permet de mettre en valeur la légèreté de la couronne $(15 \mathrm{~kg})$ et son mouvement atypique. Deux besoins sont distingués : suspendre la couronne et la faire bouger. Pour le premier point, nous avons cherché à quel(s) point(s) suspendre la couronne. Pour cela, nous avons cherché quels points étaient fixes tout au long de la rotation. Un seul l'est : le point central, mais il n'est pas matérialisé. Nous avons donc cherché à quantifier et qualifier les déplacements de points significatifs de la couronne, afin de proposer un système de suspension adapté. Une étude du modèle sur Catia nous a permis de voir que les extrémités basses des liaisons entre deux mécanismes accusaient un différentiel de $8 \mathrm{~cm}$ en hauteur, et moins de $2 \mathrm{~cm}$ dans le plan qui passe par ce point et l'axe central de la couronne. Les points hauts accusent eux un différentiel de plus de $10 \mathrm{~cm}$ en hauteur et de plus de $4 \mathrm{~cm}$ dans le plan explicité ci-dessus (figure 7).
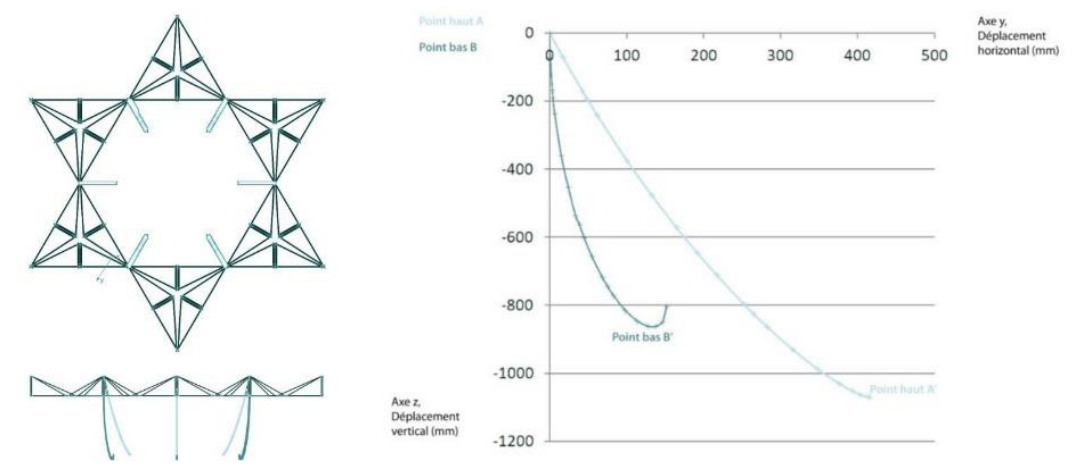

Figure 7. (a) Vue en plan et en élévation des mouvements des points hauts et bas des liaisons, (b) Graphique comparatif des mouvements des points hauts et bas pendant la rotation.

Les milieux de ces liaisons ne sont pas fixes. Chacune des six liaisons entre deux mécanismes de Bricard est donc suspendue par un câble glissé dans le trou de la barre centrale et bloqué à ses deux extrémités. Le câble peut coulisser librement dans la poulie à laquelle il est suspendu afin d'accuser le changement de position des arêtes (figure 8a). Le même dispositif de câbles est installé sur les angles libres de chaque mécanisme de Bricard, mais il sert cette fois à provoquer la rotation de la couronne (figure $8 \mathrm{~b}$ ).
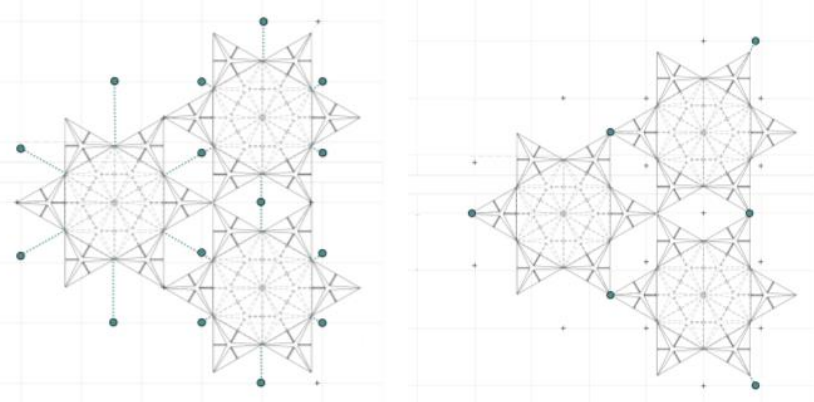

Figure 8. (a) Implantation des suspentes, (b) Implantation des robots enrouleurs.

Chaque point de suspente au plafond est situé sur la droite reliant l'arête qu'il suspend et le centre de la couronne. Vis-à-vis de la précision des accroches, cette contrainte est suffisante pour assurer le bon fonctionnement de la rotation. Il n'est ainsi pas nécessaire d'installer tous les points à équidistance du centre de la couronne, ce qui permet une grande adaptation des points d'accroches au site, ici une grille de rails (figure 9). 

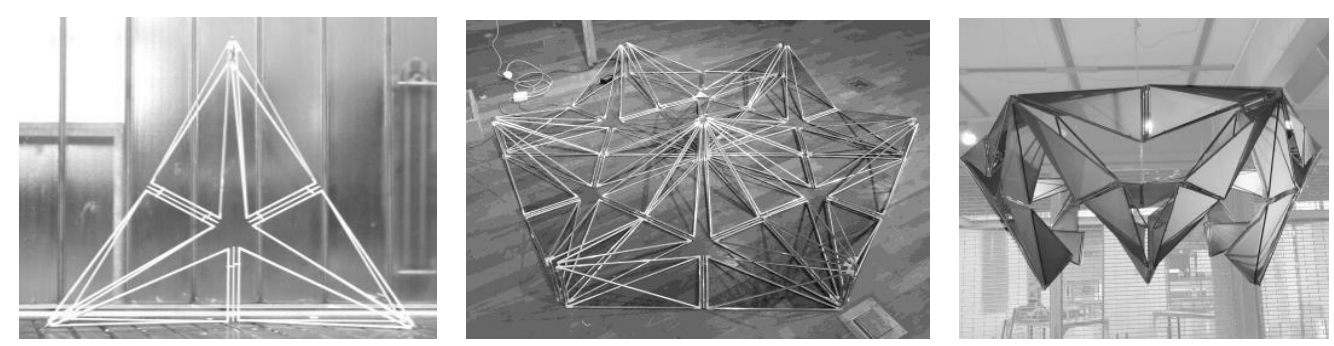

Figure 9. Photographies de l'installation de Kinésis

\section{Conclusion}

Les mécanismes de Bricard, le threefold-symmetric principalement, ont un intérêt à la fois architectural et structurel grâce à leur géométrie particulière et leur unique degré de liberté. Plusieurs propositions sont explorées pour bloquer ce degré de liberté : transformer une liaison rotoïde en encastrement, fixer physiquement la position de plusieurs points, ou encore bloquer physiquement la distance entre deux points. Plusieurs facteurs sont à prendre en compte pour la conception de mécanismes de Bricard, tel que l'usage, les jonctions ou les matériaux. Ces choix sont par ailleurs liés : dans notre cas, les tubes en aluminium sont appropriés à une utilisation en structure suspendue. Les tubes ne reprennent en effet pas de compression pure, tandis que la structure a besoin d'être le plus légère possible. Des conceptions avec des faces de tétraèdres rigides, comme le robot HexaMorph, sont adaptées pour une disposition du mécanisme au sol, où ces faces reprennent alors de la compression. D'autres matériaux restent encore à être explorés, comme les pneumatiques. De nombreuses utilisations peuvent être trouvées pour ce mécanisme en design structurel.

\section{Bibliographie}

Baker, J. E. (1980). An analysis of the Bricard linkages, Mechanism and Machine Theory, Pergamon Press, vol. 15, 267-286.

Bennett, G.T. (1903). A new mechanism, in Engineering 76, 777-778.

Bricard, R. (1897). Mémoire sur la théorie de l'octaèdre articulé, Journal de mathématiques pures et appliquées $5^{\mathrm{e}}$ série, tome 3, 113-148.

Chen, Y. (2003). Design of Structural Mechanisms, thèse de doctorat.

Chen, Y., You, Z., \& Tarnai, T. (2005). Threefold-symmetric Bricard linkages for deployable structures, International Journal of Solids and Structures, 42(8), pp. 22872301. doi: 10.1016/j.ijsolstr.2004.09.014.

Crawford, R. F., Preiswerk, P. R., \& Hedgepeth, J. M. (1975). Spoked wheels to deploy large surfaces in space: weight estimates for solar arrays. Washington, D.C.: National Aeronautics and Space Administration.

Gan, W., \& Pellegrino, S. (2003). Closed-Loop Deployable Structures, in 44th AIAA/ASME/ASCE/AHS/ASC Structures, Structural Dynamics, and Materials Conference. Reston, Virginia: American Institute of Aeronautics and Astronautics. doi: $10.2514 / 6.2003-1450$.

Gao, W., Ramani, K., Cipra, R. J. and Siegmund, T. (2013). Kinetogami: A Reconfigurable, Combinatorial, and Printable Sheet Folding, Journal of Mechanical Design, 135(11), p. 111009. doi: 10.1115/1.4025506.

Gao, W., Huo, K., Seehra, J. S., Ramani, K. and Cipra, R. J. (2014). HexaMorph: A reconfigurable and foldable hexapod robot inspired by origami, in 2014 IEEE/RSJ 
International Conference on Intelligent Robots and Systems. IEEE, pp. 4598-4604. doi: 10.1109/IROS.2014.6943214.

Luo, Y.Z., Yu, Y., \& Liu, J.Y. (2007). A retractable structure based on Bricard linkages and rotating rings of tetrahedral. International Journal of Solids and Structures, 45, 620630. 